$\mathbb{T}$ periodica polytechnica

$$
\begin{array}{r}
\text { Chemical Engineering } \\
56 / 1(2012) 3 \\
37
\end{array}
$$

doi: 10.3311/pp.ch.2012-1.01

web: http://www.pp.bme.hu/ch

(c) Periodica Polytechnica 2012

RESEARCH ARTICLE

\section{Synthesis of new potential UV-filters}

\author{
Renáta Farkas / Mercédesz Tőrincsi / Pál Kolonits / Lajos Novák
}

Received 2012-04-03, accepted 2012-04-25

\begin{abstract}
Three new silylated 2-(2'-hydroxyphenyl)benzotriazole derivatives were prepared. Starting with the easily available simple 2-(2'-hydroxyphenyl)benzotriazole, the target compounds were synthesized by a stepwise synthetic protocol, namely alkylation, thermal rearrangement and silylation.
\end{abstract}

\section{Keywords}

Benzotriazoles $\cdot$ rearrangement $\cdot$ silyl derivatives $\cdot U V$-filters

\section{Acknowledgement}

The financial support for this investigation provided by ISDIN (Barcelona, Spain) is greatly appreciated.

\section{Renáta Farkas}

Mercédesz Tőrincsi

Pál Kolonits

Department of Organic Chemistry and Technology, BME, Budapest H-1111 Szent Gellért tér 4, Hungary

\section{Lajos Novák}

Department of Organic Chemistry and Technology, BME, Budapest H-1111 Szent Gellért tér 4, Hungary

e-mail:1novak@mail.bme.hu

\section{Introduction}

As a result of the thinning of the protective ozone layer, the exposure to ultraviolet light (UV) is increasing worldwide. UV light, which is approximately $90 \%$ of this radiation, can pass through window glass, penetrates into the dermis, and may cause tanning, wrinkling, and skin cancer. Malignant melanoma is the most harmful of all skin cancers. It has been increasing faster than any other cancer and regarding the number of cases it has more than doubled in the last 5 years. Therefore, protection against the UV light has been growing and is of crucial significance [1]-[7].

A number of molecules are employed as UV light protecting agents. Among them compounds having intramolecular $\mathrm{H}$-bond are strong UV absorbers and show proper photostability. 2-(2'Hydroxyphenyl)benzotriazole derivatives (e.g. Mexoryl XL) are widely used as UV stabilizers. In these molecules the photoinduced excited state returns to the ground state by a proton transfer and rapid non-radiative dissipation of the harmful UV energy $\left(\mathrm{S}_{1} \rightarrow \mathrm{S}_{1}^{\prime} \rightarrow \mathrm{S}_{0}^{\prime} \rightarrow \mathrm{S}_{0}\right)$ [8]-[13].

Recently, we have described papers about the synthesis of new silylated hydroxyphenyl-benzotriazole derivatives. Some of them had excellent UVA-filter activity and high photostability [14]. Following this research, we have prepared three new hydroxyphenyl-benzotriazole derivatives with substituents on the benzotriazole moiety and/or on the phenyl group. The synthesis followed is shown in the Fig. 1

\section{Results and discussion}

Earlier we had elaborated a new and economical method for the synthesis of 2-(2'-hydroxyphenyl)benzotriazole derivatives (Fig. 1) and here we used its potential for the preparation of these starting materials [15, 16]. Compound 1a was then treated with 2-(chloromethyl)prop-1-ene in the presence of $\mathrm{KI}$ and $\mathrm{K}_{2} \mathrm{CO}_{3}$ to afford ether $\mathbf{2 a}$, in good yield. The ${ }^{1} \mathrm{H}$ and ${ }^{13} \mathrm{C}$ NMR spectra of $\mathbf{2 a}$ showed the typical pattern of a benzo $[d][1,2,3]$ triazole skeleton where two pairs of orthocoupled aromatic protons at $\left(\delta_{7.42}\right.$ and $\left.\delta_{7.96}\right)$ were assignable to $\mathrm{C}_{4}-\mathrm{H}$ and $\mathrm{C}_{5}-\mathrm{H}$, (or $\mathrm{C}_{6}-\mathrm{H}$ and $\mathrm{C}_{7}-\mathrm{H}$ ), respectively. Furthermore, the ${ }^{1} \mathrm{H}$ NMR spectrum revealed the presence of a 2 - 
<smiles>[X]c1ccc(O)c(-n2nc3ccc([R])cc3n2)c1</smiles>

1

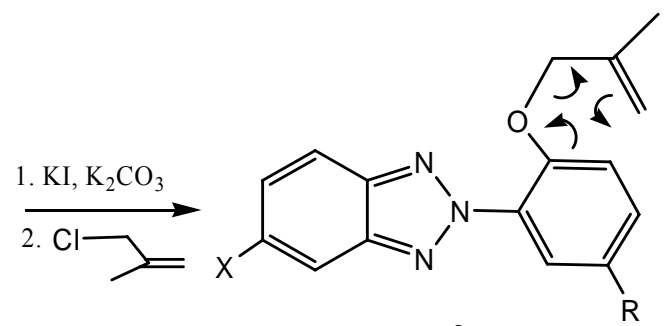

2 (i) $[3,3]$ - sigmatropic rearrangement

(ii) proton shift<smiles>[R]c1cc(CC(=C)C[SiH](C)O[Si](C)(C)C)c(O)c(-n2nc3ccc([X])cc3n2)c1</smiles>

3

4

Fig. 1.

methyl-2-propenyloxy group $\left(\delta_{4.47}, \delta_{4.86}\right.$, and $\left.\delta_{4.95}\right)$, and a cyclohexylphenyl moiety. Thermal rearrangement of compounds 2a in boiling $N, N$-diethylbenzeneamine afforded the wanted intermediate 3a, in excellent yield. In the ${ }^{1} \mathrm{H}$ spectrum the signal at $\left(\delta_{11.42}, \mathrm{OH}\right)$ together with the other part of spectra justified the structure of 3a. This compound was then silylated with heptamethyltrisiloxane in the presence of Karlstedt catalyst [17] to furnish the target compounds $\mathbf{4 a}$, in good yield. The structure of compound $\mathbf{4 a}$ was also justified by its spectra. For instance, both ${ }^{1} \mathrm{H}$ and ${ }^{13} \mathrm{C}$ NMR spectra showed the characteristic signals of the benzotriazole and cyclohexylphenyl skeleton, the hydroxyl group $\left(\delta_{11.34}\right)$, and the heptamethyl-disiloxanyl moiety $\left[\delta_{0.08}\left(\mathrm{Si}-\mathrm{CH}_{3}\right)\right.$ and $\left.\delta_{0,11}\left(18 \mathrm{H}, 6 \mathrm{CH}_{3}\right)\right]$.

The reaction of the chloro compound $\mathbf{1 b}$ with 2(chloromethyl)prop-1-ene gave the ether $\mathbf{2 b}$, which was thermally rearranged. The product $\mathbf{3 b}$ was then silylated with heptamethyltrisiloxane in the presence of Karlstedt catalyst to afford compound $\mathbf{4 b}$, in an overall yield $44.3 \%$.

Likewise, the methoxy analog $\mathbf{4 c}$ was prepared with the alkylation of 1c followed by rearrangement and silylation. Here, the overall yield of the three steps was lower (15.6\%).

\section{Conclusion}

We have elaborated a straightforward method for the preparation of three potential UV-filters. The synthesis of these compounds $4 \mathbf{4 a - c}$ was achieved in three steps starting with the easily available benzotriazole derivatives 1a-c, in good or acceptable overall yield. The UV absorption and photostability of these new compounds will be published in due course.

\section{Experimental}

All solvents were used as received from commercial vendors and no further attempts were made to purify or dry them. Melting points were determined on a Büchi apparatus and are uncorrected. IR spectra were recorded on a Bruker Alpha FT Spectrophotometer. ${ }^{1} \mathrm{H}$ NMR and ${ }^{13} \mathrm{C}$ NMR were recorded on a Bruker DRX-500 spectrometer operating at $500 \mathrm{MHz}$ and 125 $\mathrm{MHz}$, respectively. All NMR spectra are reported in ppm relative to TMS, used as an internal standard. The ${ }^{1} \mathrm{H}$ and ${ }^{13} \mathrm{C}$ NMR signals were assigned on the bases of ATP, COSY, HMQC, and HMBC experiences. Merck precoated silica gel $60 \mathrm{~F}_{254}$ plates were used for TLC and Kieselgel 60 for column chromatography using a solution of hexane-EtOAc $(5: 0.2)$ as eluent. Elemental analyses for $\mathrm{C}, \mathrm{H}, \mathrm{N}$ agreed favorably with calculated values.

\subsection{General procedure for the preparation of compounds 2a-c}

Compound 1 (23.6 mmol), $\mathrm{KI}(3.9 \mathrm{~g}, 23.6 \mathrm{mmol})$, and $\mathrm{K}_{2} \mathrm{CO}_{3}$ $(3.2 \mathrm{~g}, 23.6 \mathrm{mmol})$ were mixed in butan-2-one $(100 \mathrm{ml})$ and after adding 2-(chloromethyl)prop-1-ene (5.0 g, $5.6 \mathrm{ml}, 23.8 \mathrm{mmol}$ ) the resulting mixture was heated under reflux for $4 \mathrm{~h}$. After cooling, the precipitate was filtered off and the solvent was evaporated in vacuo. The residue was purified by column chromatography.

The following products were thus prepared:

2-[2-(2-Methylallyloxy)-5-cyclohexylphenyl)- $2 \mathrm{H}$ benzo[d][1,2,3]triazole $(\mathbf{2 a})$

Yield: $60 \%$, yellow oil; TLC: $R_{f}=0.74$ (hexane-acetone 5:2); IR (KBr): 2950, 1520, 1475, 1413, $1255 \mathrm{~cm}^{-1} ;{ }^{1} \mathrm{H}-\mathrm{NMR}$ 
$\left(\mathrm{CDCl}_{3}\right): 1.24\left(\mathrm{~m}, 1 \mathrm{H}, \mathrm{C}_{4}{ }^{\prime} \mathrm{H}\right), 1.35\left(\mathrm{~m}, 2 \mathrm{H}, \mathrm{C}_{3}\right.$ "'-H and $\mathrm{C}_{5}$ "$\mathrm{H}), 1.42\left(\mathrm{~m}, 2 \mathrm{H}, \mathrm{C}_{2},-\mathrm{H}\right.$ and $\left.\mathrm{C}_{6},-\mathrm{H}\right), 1.67$ (s, 3H, $\left.\mathrm{CH}_{3}\right), 1.72$ (m, 1H, C 4 "-H), 1.83 (m, 2H, C 3 "- $-\mathrm{H}$ and $\mathrm{C}_{5}$ "- $\mathrm{H}$ ), 1.91 (m, 2H, $\mathrm{C}_{2}$ " $-\mathrm{H}$ and $\mathrm{C}_{6}$ "-H), $2.53\left(\mathrm{~m}, 1 \mathrm{H}, \mathrm{C}_{1}\right.$ "-H), 4.47 (s, 2H, $\left.\mathrm{OCH}_{2}\right)$, $4.86\left(\mathrm{~s}, 1 \mathrm{H},=\mathrm{CH}_{2}\right), 4.95\left(\mathrm{~s}, 1 \mathrm{H},=\mathrm{CH}_{2}\right), 7.04(\mathrm{~d}, J=8.6 \mathrm{~Hz}$, $\left.\mathrm{C}_{3},-\mathrm{H}\right), 7.29$ (dd, $J=8.6$ and $\left.1.9 \mathrm{~Hz}, 1 \mathrm{H}, \mathrm{C}_{4},-\mathrm{H}\right), 7.42$ (dd, $J=6.6$ and $3.0 \mathrm{~Hz}, 2 \mathrm{H}, \mathrm{C}_{5}-\mathrm{H}$ and $\left.\mathrm{C}_{6}-\mathrm{H}\right), 7.52(\mathrm{~d}, J=1.9 \mathrm{~Hz}$, $\left.1 \mathrm{H}, \mathrm{C}_{6},-\mathrm{H}\right), 7.96\left(\mathrm{dd}, J=6.6\right.$ and $3.0 \mathrm{~Hz}, 2 \mathrm{H}, \mathrm{C}_{4}-\mathrm{H}$ and $\mathrm{C}_{7}-$ $\mathrm{H}) ;{ }^{13} \mathrm{C}-\mathrm{NMR}\left(\mathrm{CDCl}_{3}\right): 19.17 \mathrm{CH}_{3}, 26.05$ (C-4"), 26.80 (C-3" and C-5"), 34.48 (C-2" and C-6"), 43.55 (C-1"), $72.84\left(\mathrm{OCH}_{2}\right)$, $112.66(=\mathrm{C}), 114.41$ (C-3'), 118.40 (C-4 and C-7), 125.69 (C6'), 126.65 (C-5 and C-6), 129.18 (C-4'), 130.44 (C-1'), 140.17 $(\mathrm{C}=), 141.04$ (C-3'), 144.75 (C-7a), 150.69 (C-3a). Anal. Calcd for $\mathrm{C}_{22} \mathrm{H}_{25} \mathrm{~N}_{3} \mathrm{O}$ : C, 76.05; H, 7.25; N, 12.09. Found: C,76.17; $\mathrm{H}, 7.02 ; \mathrm{N}, 11.94$.

5-Chloro-2-\{4-[2-methyl-2-propen-1-yl]-3-biphenylyl $\}-2 H$ 1,2,3-benzotriazole (2b).

Yield: $74 \%$, light yellow crystals; m.p.: $107-108{ }^{\circ}$ C; TLC: $R_{f}=0.65$ (hexane-acetone 5:2); IR (KBr): 1489, 1290, 12.61, $909 \mathrm{~cm}^{-1} ;{ }^{1} \mathrm{H}-\mathrm{NMR}\left(\mathrm{CDCl}_{3}\right): 1.71\left(\mathrm{~s}, 3 \mathrm{H}, \mathrm{CH}_{3}\right), 4.55(\mathrm{~s}, 2 \mathrm{H}$, $\left.\mathrm{OCH}_{2}\right), 4.91\left(\mathrm{~s}, 1 \mathrm{H},=\mathrm{CH}_{2}\right), 5.00\left(\mathrm{~s}, 1 \mathrm{H},=\mathrm{CH}_{2}\right), 7.19(\mathrm{~d}, J=$ $\left.8.7 \mathrm{~Hz}, 1 \mathrm{H}, \mathrm{C}_{5},-\mathrm{H}\right), 7.34$ (t, J= $\left.7.5 \mathrm{~Hz}, \mathrm{C}_{10},-\mathrm{H}\right), 7.39$ (dd, $J=9.1$ and $\left.1.5 \mathrm{~Hz}, 1 \mathrm{H}, \mathrm{C}_{6}-\mathrm{H}\right), 7.43\left(\mathrm{t}, J=7.5 \mathrm{~Hz}, 2 \mathrm{H}, \mathrm{C}_{9}\right.$, $\mathrm{H}$ and $\left.\mathrm{C}_{11},-\mathrm{H}\right), 7.59\left(\mathrm{~d}, J=8.1 \mathrm{~Hz}, 2 \mathrm{H}, \mathrm{C}_{8},-\mathrm{H}\right.$ and $\left.\mathrm{C}_{12},-\mathrm{H}\right)$, $7.70\left(\mathrm{dd}, J=8.7\right.$ and $\left.2.1 \mathrm{~Hz}, 1 \mathrm{H}, \mathrm{C}_{6},-\mathrm{H}\right), 7.92(\mathrm{~d}, J=9.1 \mathrm{~Hz}$, $\left.1 \mathrm{H}, \mathrm{C}_{7}-\mathrm{H}\right), 7.93$ (s, 1H, C,$\left.-\mathrm{H}\right), 7.97$ (s, $\left.1 \mathrm{H} \mathrm{C}-\mathrm{H}\right) ;{ }^{13} \mathrm{C}-\mathrm{NMR}$ $\left(\mathrm{CDCl}_{3}\right): 19.17\left(\mathrm{CH}_{3}\right), 72.78\left(\mathrm{OCH}_{2}\right), 112.99(=\mathrm{C}), 114.71(\mathrm{C}-$ 5'), 117.43 (C-4), 119.67 (C-7), 125.98 (C-2'), 126.81 (C-8' and C-12'), 127.44 (C-10'), 128.46 (C-6), 128.90 (C-9' and C11'), 129.51 (C-6'), 130.61 (C-3'), 132.67 (C-5), 134.36 (C1'), 139.22 (C-7'), 139.80 (C=), 143.32 (C-7a), 145.13 (C-3a), 151.90 (C-4'). Anal. Calcd for $\mathrm{C}_{22} \mathrm{H}_{18} \mathrm{ClN}_{3} \mathrm{O}: \mathrm{C}, 70.30 ; \mathrm{H}$, 4.83; N, 11.18. Found: C, 70.12; H, 5.04; N, 10.96.

5-Methoxy-2-\{ 4-[2-methyl-2-propen-1-yl]-3-biphenylyl $\}$ 2H-1,2,3-benzotriazole (2c).

Yield: $70 \%$, light yellow crystals; m.p.: $115-116{ }^{\circ} \mathrm{C}$; TLC: $R_{f}=0.44$ (hexane-EtOAc 4:1); ${ }^{1} \mathrm{H}-\mathrm{NMR}\left(\mathrm{CDCl}_{3}\right): 1.71(\mathrm{~s}, 3 \mathrm{H}$, $\left.\mathrm{CH}_{3}\right), 3.91\left(\mathrm{~s}, 3 \mathrm{H}, \mathrm{OCH}_{3}\right), 4.55\left(\mathrm{~s}, 2 \mathrm{H}, \mathrm{OCH}_{2}\right), 4.91(\mathrm{~s}, 1 \mathrm{H}$, $\left.=\mathrm{CH}_{2}\right), 5.01\left(\mathrm{~s}, 1 \mathrm{H},=\mathrm{CH}_{2}\right), 7.34\left(\mathrm{t}, J=7.5 \mathrm{~Hz}, 1 \mathrm{H}, \mathrm{C}_{10},-\mathrm{H}\right)$, $7.39\left(\mathrm{dd}, J=9.1\right.$ and $\left.1.5 \mathrm{~Hz}, 1 \mathrm{H}, \mathrm{C}_{6}-\mathrm{H}\right), 7.43(\mathrm{t}, J=7.5 \mathrm{~Hz}, 2 \mathrm{H}$, $\mathrm{C}_{9},-\mathrm{H}$ and $\left.\mathrm{C}_{11},-\mathrm{H}\right), 7.59\left(\mathrm{~d}, J=8.1 \mathrm{~Hz}, 2 \mathrm{H}, \mathrm{C}_{8},-\mathrm{H}\right.$ and $\left.\mathrm{C}_{12},-\mathrm{H}\right)$, $7.70\left(\mathrm{dd}, J=8.7\right.$ and $\left.2.1 \mathrm{~Hz}, 1 \mathrm{H}, \mathrm{C}_{6},-\mathrm{H}\right), 7.92(\mathrm{~d}, J=9.1 \mathrm{~Hz}$, $\left.1 \mathrm{H}, \mathrm{C}_{7}-\mathrm{H}\right), 7.93$ (s, 1H, C 3 - $\left.\mathrm{H}\right), 7.97$ (s, 1H, C $\left.4-\mathrm{H}\right) ;{ }^{13} \mathrm{C}-\mathrm{NMR}$ $\left(\mathrm{CDCl}_{3}\right): 19.17\left(\mathrm{CH}_{3}\right), 55.54\left(\mathrm{OCH}_{3}\right), 72.76\left(\mathrm{OCH}_{2}\right), 112.99$ (=C), 117.43 (C-4), 119.67 (C-7), 125.98 (C-2'), 126.81 (C8' and C-12'), 127.44 (C-10'), 128.90 (C-9' and C-11'), 129.51 (C-6'), 130.61 (C-3'), 133.62 (C-1'), 134.36 (C-5'), 139.22 (C7'), 139.80 (C=), 143.32 (C-7a), 145.13 (C-3a), 151.90 (C-4'). Anal. Calcd for $\mathrm{C}_{23} \mathrm{H}_{21} \mathrm{~N}_{3} \mathrm{O}_{2}$ : C, 74.37; H, 5.70; N, 11.31. Found: C, 74.21; H, 5.50; N, 11.11,
4.2 General procedure for the synthesis of $3 \mathbf{a}-\mathbf{c}$ by thermal rearrangement

A solution of compound $3(30 \mathrm{mmol})$ in $N, N$ diethylbenzeneamine $(50 \mathrm{ml})$ was heated under reflux for $4 \mathrm{~h}$. After cooling, the mixture was treated with aqueous $\mathrm{HCl}$ $(15 \%, 50 \mathrm{ml})$ and then extracted three times with $\mathrm{CH}_{2} \mathrm{Cl}_{2}(50$ $\mathrm{ml}$ each). The combined organic extracts were dried $\left(\mathrm{MgSO}_{4}\right)$, and concentrated to give a solid residue which was crystallized from hexane.

The following products were thus prepared:

2-(2H-1,2,3 benzotriazol-2-yl)-4-cyclohexyl-6-(2-methyl-2propen-1-yl)phenol (3a).

Yield: $94 \%$, white crystals; m.p.: $125-127^{\circ}$ C; TLC: $R_{f}=0.9$ (hexane-acetone 5:2); ${ }^{1} \mathrm{H}-\mathrm{NMR}\left(\mathrm{CDCl}_{3}\right): 1.29\left(\mathrm{~m}, 1 \mathrm{H}, \mathrm{C}_{4},-\mathrm{H}\right)$, $1.40\left(\mathrm{~m}, 2 \mathrm{H}, \mathrm{C}_{3}\right.$ "- $\mathrm{H}$ and $\mathrm{C}_{5}$ "'-H), $1.47\left(\mathrm{~m}, 2 \mathrm{H}, \mathrm{C}_{2}\right.$ "- $\mathrm{H}$ and $\mathrm{C}_{6}$ "$\mathrm{H}), 1.76\left(\mathrm{~d}, J=12.5 \mathrm{~Hz}, 1 \mathrm{H}, \mathrm{C}_{4}\right.$ ",-H), $2.55\left(\mathrm{~m}, 1 \mathrm{H}, \mathrm{C}_{1}\right.$ ",-H), 3.49 (s, 2H, $\left.\mathrm{CH}_{2}\right), 4.74\left(\mathrm{~s}, 1 \mathrm{H},=\mathrm{CH}_{2}\right), 4.86\left(\mathrm{~s}, 1 \mathrm{H},=\mathrm{CH}_{2}\right), 7.09(\mathrm{~d}$, $\left.J=1.7 \mathrm{~Hz}, 1 \mathrm{H}, \mathrm{C}_{5}-\mathrm{H}\right), 7.46\left(\mathrm{dd}, J=6.5\right.$ and $3.0 \mathrm{~Hz}, 2 \mathrm{H}, \mathrm{C}_{5},-\mathrm{H}$ and $\left.\mathrm{C}_{6},-\mathrm{H}\right), 7.92\left(\mathrm{dd}, J=6.5\right.$ and $3.0 \mathrm{~Hz}, 2 \mathrm{H}, \mathrm{C}_{4},-\mathrm{H}$ and $\left.\mathrm{C}_{7},-\mathrm{H}\right)$, $8.13\left(\mathrm{~d}, J=1.9 \mathrm{~Hz}, 1 \mathrm{H}, \mathrm{C}_{3}-\mathrm{H}\right), 11.42(\mathrm{~s}, 1 \mathrm{H}, \mathrm{OH}) ;{ }^{13} \mathrm{C}-\mathrm{NMR}$ $\left(\mathrm{CDCl}_{3}\right): 22.55\left(\mathrm{CH}_{3}\right), 26.11(\mathrm{C}-4$ "), $26.90(\mathrm{C}-3$ " and C-5"), 34.60 (C-2" and C-6"), $38.05\left(\mathrm{CH}_{2}\right), 43.78$ (C-1"), 111.58 (=C), 117.19 (C-3), 117.61 (C-4' and C-7'), 124.88 (C-2), 127.53 (C5' and C-6'), 129.26 (C-6), 130.24 (C-5), 139.39 (C-4), 142.75 (C-3a' and C-7a'), $144.56(\mathrm{C}=), 146.04$ (C-1). Anal. Calcd for $\mathrm{C}_{22} \mathrm{H}_{25} \mathrm{~N}_{3} \mathrm{O}$ : C, 76.05; H, 7.25; N, 12.09. Found: C,75.93; H, 7.14; N, 11.96.

3-(5-Chloro-2H-1,2,3-benzotriazol-2-yl)-5-(2-methyl-2propen-1-yl)-4-biphenylol (3b).

Yield: $88 \%$, light yellow crystals; m.p.: $138-139{ }^{\circ} \mathrm{C}$; TLC: $R_{f}=0.87$ (hexane-acetone 5:2); ${ }^{1} \mathrm{H}-\mathrm{NMR}\left(\mathrm{CDCl}_{3}\right): 1.83(\mathrm{~s}$, $\left.3 \mathrm{H}, \mathrm{CH}_{3}\right), 3.56\left(\mathrm{~s}, 2 \mathrm{H}, \mathrm{CH}_{2}\right), 4.79\left(\mathrm{~s}, 1 \mathrm{H},=\mathrm{CH}_{2}\right), 4.89(\mathrm{~s}, 1 \mathrm{H}$, $\left.=\mathrm{CH}_{2}\right), 7.35\left(\mathrm{t}, J=7.4 \mathrm{~Hz}, 1 \mathrm{H}, \mathrm{C}_{10}-\mathrm{H}\right), 7.42(\mathrm{dd}, J=9.2$ and $\left.1.6 \mathrm{~Hz}, 1 \mathrm{H}, \mathrm{C}_{6},-\mathrm{H}\right), 7.44\left(\mathrm{t}, J=7.5 \mathrm{~Hz}, 2 \mathrm{H}, \mathrm{C}_{9}-\mathrm{H}\right.$ and $\left.\mathrm{C}_{11}-\mathrm{H}\right)$, $7.50\left(\mathrm{~d}, J=1.8 \mathrm{~Hz}, 1 \mathrm{H}, \mathrm{C}_{6}-\mathrm{H}\right), 7.65\left(\mathrm{~d}, J=7.5 \mathrm{~Hz}, 2 \mathrm{H}, \mathrm{C}_{8}-\mathrm{H}\right.$ and $\left.\mathrm{C}_{12}-\mathrm{H}\right), 7.87\left(\mathrm{~d}, J=9.0 \mathrm{~Hz}, 1 \mathrm{H}, \mathrm{C}_{7},-\mathrm{H}\right), 7.91(\mathrm{~d}, J=1.2$ $\left.\mathrm{Hz}, 1 \mathrm{H}, \mathrm{C}_{4},-\mathrm{H}\right), 8.51$ (d, J=2.2 Hz, 1H, C $\left.2-\mathrm{H}\right), 11.40$ (s, $1 \mathrm{H}$, $\mathrm{OH}) ;{ }^{13} \mathrm{C}-\mathrm{NMR}\left(\mathrm{CDCl}_{3}\right): 22.56\left(\mathrm{CH}_{3}\right), 38.04\left(\mathrm{CH}_{2}\right), 111.98$ (=C), 116.67 (C-4'), 117.76 (C-2), 118.82 (C-7'), 125.20 (C-3), 126.80 (C-8 and C-12), 127.22 (C-10), 128.85 (C-9 and C-11), 129.32 (C-3), 130.23 (C-5), 130.33 (C-6), 132.80 (C-1), 133.67 (C-5'), 139.81 (C-7), 141.33 (C-7a'), 143.15 (C-3a'), 144.15 $(\mathrm{C}=), 147.46$ (C-4). Anal. Calcd for $\mathrm{C}_{22} \mathrm{H}_{18} \mathrm{ClN}_{3} \mathrm{O}$ : C, 70.30; H, 4.83; N, 11.18. Found: C, 70.08; H, 4.64; N, 11.06.

3-(5-Methoxy-2H-1,2,3-benzotriazol-2-yl)-5-(2-methyl-2propen-1-yl)-4-biphenylol (3c).

Yield: $50 \%$, yellow crystals; m.p.: $125-126{ }^{\circ} \mathrm{C}$; TLC: $R_{f}=0.75$ (hexane-EtOAc 4:1); ${ }^{1} \mathrm{H}-\mathrm{NMR}\left(\mathrm{CDCl}_{3}\right): 1.84(\mathrm{~s}$, $\left.3 \mathrm{H}, \mathrm{CH}_{3}\right), 3.56\left(\mathrm{~s}, 2 \mathrm{H}, \mathrm{CH}_{2}\right), 3.91\left(\mathrm{~s}, 3 \mathrm{H}, \mathrm{OCH}_{3}\right), 4.80(\mathrm{~s}$, $\left.1 \mathrm{H},=\mathrm{CH}_{2}\right), 4.88\left(\mathrm{~s}, 1 \mathrm{H},=\mathrm{CH}_{2}\right), 7.11\left(\mathrm{C}_{4},-\mathrm{H}\right), 7.14(\mathrm{~d}, J=9.2$ $\left.\mathrm{Hz}, 1 \mathrm{H}, \mathrm{C}_{6},-\mathrm{H}\right), 7.34\left(\mathrm{t}, J=7.5 \mathrm{~Hz}, 1 \mathrm{H}, \mathrm{C}_{10}-\mathrm{H}\right), 7.43$ (t, $J=$ $7.8 \mathrm{~Hz}, 2 \mathrm{H}, \mathrm{C}_{9}-\mathrm{H}$ and $\left.\mathrm{C}_{11}-\mathrm{H}\right), 7.46\left(\mathrm{~s}, 1 \mathrm{H}, \mathrm{C}_{6}-\mathrm{H}\right), 7.66(\mathrm{~d}, J=$ 
$7.5 \mathrm{~Hz}, 2 \mathrm{H}, \mathrm{C}_{8}-\mathrm{H}$ and $\left.\mathrm{C}_{12}-\mathrm{H}\right), 7.77\left(\mathrm{~d}, J=9.2 \mathrm{~Hz}, 1 \mathrm{H}, \mathrm{C}_{7}\right.$ $\mathrm{H}), 8.48$ (s, $\left.1 \mathrm{H}, \mathrm{C}_{2}-\mathrm{H}\right), 11.60(\mathrm{~s}, 1 \mathrm{H}, \mathrm{OH}) ;{ }^{13} \mathrm{C}-\mathrm{NMR}\left(\mathrm{CDCl}_{3}\right)$ : $22.57\left(\mathrm{CH}_{3}\right), 38.07\left(\mathrm{CH}_{2}\right), 55.67\left(\mathrm{OCH}_{3}\right), 94.21\left(\mathrm{C}-4{ }^{\prime}\right), 111.88$ (=C), 117.33 (C-2), 118.45 (C-7'), 122.91 (C-6'), 126.82 (C-8 and C-12), 127.07 (C-10), 128.79 (C-9 and C-11), 129.41 (C6), 129.94 (C-5), 132.57 (C-1), 139.00 (C-7a'), 140.08 (C-7), 143.92 (C-3a'), 144.33 (C=), 147.12 (C-4), 159.81 (C-5'). Anal. Calcd for $\mathrm{C}_{23} \mathrm{H}_{21} \mathrm{~N}_{3} \mathrm{O}_{2}$ : C, 74.37; H, 5.70; N, 11.31. Found: C, $74.11 ; \mathrm{H}, 5.53 ; \mathrm{N}, 11.15$.

\subsection{General procedure for the synthesis of compounds 4}

To a stirred solution of compound $\mathbf{3}(3 \mathrm{mmol})$ in xylene (20 $\mathrm{ml}$ ) were added 1,1,1,3.5,5,5-heptamethyltrisiloxane (1 g, 4.5 mmol) and Karlstedt catalyst (10 drops), and the resulting mixture was heated at $100{ }^{\circ} \mathrm{C}$ for $8 \mathrm{~h}$. After cooling, the solvent was evaporated in vacuo and the residue was purified by column chromatography.

The following products were thus prepared:

2-(2H-1,2,3-Benzotriazol-2-yl)-4-cyclohexyl-6-

(2-methyl-3-\{1,3,3,3-tetramethyl-1-[trimethylsilyloxy]disiloxanyl)propyl $\}$ phenol (4a).

Yield: $66 \%$, yellow oil; TLC: $R_{f}=0.78$ (hexane-acetone 5:0.1); IR (KBr): 1471, 1250, 1142, $1037 \mathrm{~cm}^{-1} ;{ }^{1} \mathrm{H}-\mathrm{NMR}$ $\left(\mathrm{CDCl}_{3}\right): 0.08\left(\mathrm{~s}, 3 \mathrm{H}, \mathrm{CH}_{3}\right), 0.11\left(\mathrm{~s}, 18 \mathrm{H}, 6 \mathrm{CH}_{3}\right), 0.51$ (dd, $J=14.8$ and $\left.9.3 \mathrm{~Hz}, 1 \mathrm{H}, \mathrm{C}_{3}, "-\mathrm{H}\right), 0.74(\mathrm{dd}, J=14.8$ and 4.5 $\mathrm{Hz}, 1 \mathrm{H}, \mathrm{C}_{3}$ ", $\left.-\mathrm{H}\right), 1.02$ (d, J=6.6 Hz, 3H, C 3 "-H), 1.33 (m, 1H, $\mathrm{C}_{4}$ ",-H), 1.44 (m, 2H, C 3 "-H and $\mathrm{C}_{5}$ ",-H), 1.52 (m, 2H, C ${ }_{2},-\mathrm{H}$ and $\left.\mathrm{C}_{6},-\mathrm{H}\right), 1.80\left(\mathrm{~m}-\mathrm{d}, J=12.5 \mathrm{~Hz}, 2 \mathrm{H}, \mathrm{C}_{4}\right.$ "'-H), 1.90 (m-d, $J=$ $12.5 \mathrm{~Hz}, 2 \mathrm{H}, \mathrm{C}_{3}$ "-H), 1.97 (m-d, $J=12.5 \mathrm{~Hz}, 2 \mathrm{H}, \mathrm{C}_{2}$ "- $\mathrm{H}$ and $\left.\mathrm{C}_{6},-\mathrm{H}\right), 2.16\left(\mathrm{~m}, 1 \mathrm{H}, \mathrm{C}_{2},{ }^{\prime},-\mathrm{H}\right), 2.58\left(\mathrm{~m}, 1 \mathrm{H}, \mathrm{C}_{1},-\mathrm{H}\right), 2.65$ (dd, $J=13.1$ and $\left.7.9 \mathrm{~Hz}, 1 \mathrm{H}, \mathrm{C}_{1},{ }^{\prime}, \mathrm{H}\right), 2.78(\mathrm{dd}, J=13.1$ and 6.3 $\left.\mathrm{Hz}, 1 \mathrm{H}, \mathrm{C}_{1},{ }^{-}-\mathrm{H}\right), 7.07$ (d, $\left.J=1.6 \mathrm{~Hz}, 1 \mathrm{H}, \mathrm{C}_{5}-\mathrm{H}\right), 7.49$ (dd, $J=$ 6.6 and $\left.3.0 \mathrm{~Hz}, 2 \mathrm{H}, \mathrm{C}_{5},-\mathrm{H}\right), 7.96(\mathrm{dd}, J=6.6$ and $3.0 \mathrm{~Hz}, 2 \mathrm{H}$, $\mathrm{C}_{4},-\mathrm{H}$ and $\left.\mathrm{C}_{7},-\mathrm{H}\right), 8.13\left(\mathrm{~d}, J=1.6 \mathrm{~Hz}, 1 \mathrm{H}, \mathrm{C}_{3}-\mathrm{H}\right), 11.34(\mathrm{~s}$, $1 \mathrm{H}, \mathrm{OH}) ;{ }^{13} \mathrm{C}-\mathrm{NMR}\left(\mathrm{CDCl}_{3}\right): 0.82\left(\mathrm{CH}_{3}\right), 1.86\left(\mathrm{CH}_{3}\right), 22.51$ $\left(\mathrm{CH}_{3}\right), 26.04$ (C-3"”), 26.12 (C-4"), 26.90 (C-5"), 29.26 (C2"), 34.62 (C-2" and C-6"), 41.22 (C-1"”), 43.78 (C-1"), 116.78 (C-3), 117.60 (C-4' and C-7'), 124.79 (C-2), 127.41 (C-5' and C-6'), 130.77 (C-5), 131.34 (C-6), 138.96 (C-4), 142.72 (C-3a and C-7a), 146.15 (C-1). Anal. Calcd for $\mathrm{C}_{29} \mathrm{H}_{47} \mathrm{~N}_{3} \mathrm{O}_{3} \mathrm{Si}_{3}$ : C, 61.00; H, 8.31; N, 7.37. Found: C, 59.89; H, 8.22; N, 7.16.

3-(5-Chloro-2H-1,2,3-benzotriazol-2-yl)-5-(2methyl-3-\{1,3,3,3-tetramethyl-1-[trimethylsilyl disiloxanyl\}propyl)-4-biphenylol (4b).

Yield: $68 \%$, yellow oil; TLC: $R_{f}=0.62$ (hexane-acetone 5:0.1); IR (KBr): 1475, 1255, 1033, $849 \mathrm{~cm}^{-1}$; ${ }^{1} \mathrm{H}-\mathrm{NMR}$ $\left(\mathrm{CDCl}_{3}\right): 0.10\left(\mathrm{~s}, 9 \mathrm{H}, 3 \mathrm{CH}_{3}\right), 0.14\left(\mathrm{~s}, 3 \mathrm{H}, \mathrm{CH}_{3}\right), 0.15(\mathrm{~s}, 3 \mathrm{H}$, $\mathrm{CH}_{3}$ ), 0.58 (dd, $J=14.8$ and $\left.9.2 \mathrm{~Hz}, 1 \mathrm{H}, \mathrm{C}_{3},-\mathrm{H}\right), 0.81(\mathrm{dd}, J=$ 14.8 and $\left.4.6 \mathrm{~Hz}, 1 \mathrm{H}, \mathrm{C}_{3},-\mathrm{H}\right), 1.08\left(\mathrm{~d}, J=6.9 \mathrm{~Hz}, 3 \mathrm{H}, \mathrm{CH}_{3}\right)$, 2.25 (m, 1H, C ${ }_{2}$ - $\left.-\mathrm{H}\right), 2.73$ (dd, $J=13.2$ and $8.3 \mathrm{~Hz}, 1 \mathrm{H}, \mathrm{C}_{1}$ ",-H), $2.90\left(\mathrm{dd}, J=13.2\right.$ and $\left.6.2 \mathrm{~Hz}, 1 \mathrm{H}, \mathrm{C}_{1},-\mathrm{H}\right), 7.40(\mathrm{t}, J=7.3,1 \mathrm{H}$, $\left.\mathrm{C}_{10}-\mathrm{H}\right), 7.44\left(\mathrm{dd}, J=9.0\right.$ and $\left.1.1 \mathrm{~Hz}, 1 \mathrm{H}, \mathrm{C}_{6},-\mathrm{H}\right), 7.50(\mathrm{~m}, 3 \mathrm{H}$, $\mathrm{C}_{6}-\mathrm{H}, \mathrm{C}_{9}-\mathrm{H}$, and $\left.\mathrm{C}_{11}-\mathrm{H}\right), 7.70\left(\mathrm{~d}, J=7.6 \mathrm{~Hz}, 2 \mathrm{H}, \mathrm{C}_{8}-\mathrm{H}\right.$ and $\mathrm{C}_{12}-$
$\mathrm{H}), 7.89\left(\mathrm{~d}, J=9.0 \mathrm{~Hz}, 1 \mathrm{H}, \mathrm{C}_{7},-\mathrm{H}\right), 7.95\left(\mathrm{~s}, 1 \mathrm{H}, \mathrm{C}_{4},-\mathrm{H}\right), 8.51(\mathrm{~d}$ $\left.J=1.8 \mathrm{~Hz}, 1 \mathrm{H}, \mathrm{C}_{2}-\mathrm{H}\right), 11.61(\mathrm{~s}, 1 \mathrm{H}, \mathrm{OH}) ;{ }^{13} \mathrm{C}-\mathrm{NMR}\left(\mathrm{CDCl}_{3}\right)$ : $0.87\left(\mathrm{CH}_{3}\right), 1.88\left(\mathrm{CH}_{3}\right), 22.48\left(\mathrm{CH}_{3}\right), 26.16(\mathrm{C}-3$ ”) $), 29.27(\mathrm{C}-$ 2”), 41.26 (C-1”), 117.34 (C-2), , 118.75 (C-7'), 125.09 (C-3), 126.76 (C-8 and C-12), 127.09 (C-10), 128.78 (C-9 and C-11), 129.14 (C-6'), 130.85 (C-6), 132.24 (C-5), 132.42 (C-1), 133.51 (C-5'), 139.96 (C-7), 141.25 (C-7a'), 143.08 (C-3a'), 147.59 (C4). Anal. Calcd for $\mathrm{C}_{29} \mathrm{H}_{40} \mathrm{ClN}_{3} \mathrm{O}_{3} \mathrm{Si}_{3}: \mathrm{C}, 58.21 ; \mathrm{H}, 6.74 ; \mathrm{N}$, 7.02. Found: C, 57.94; H, 6.52; N, 7.15.

\section{3-(5-Methoxy-2H-1,2,3-benzotriazol-2-yl)-5-(2-methyl-3-}

$\{1,3,3,3$-tetramethyl-1-[trimethylsilyl oxy]disiloxanyl $\}$ propyl)4-biphenylol (4c).

Yield: $45 \%$, yellow crystals; m.p.: $56-59^{\circ} \mathrm{C}$; TLC: $R_{f}=0.52$ (hexane-EtOAc 5:2); ${ }^{1} \mathrm{H}-\mathrm{NMR}\left(\mathrm{CDCl}_{3}\right): 0.11\left(\mathrm{~s}, 3 \mathrm{H}, \mathrm{CH}_{3}\right)$, $0.12\left(\mathrm{~s}, 9 \mathrm{H}, 3 \mathrm{CH}_{3}\right), 0.13\left(\mathrm{~s}, 9 \mathrm{H}, 3 \mathrm{CH}_{3}\right), 0.57(\mathrm{dd}, J=14.8$ and $\left.9.2 \mathrm{~Hz}, 1 \mathrm{H}, \mathrm{C}_{3},{ }_{-} \mathrm{H}\right), 0.80\left(\mathrm{dd}, J=14.8\right.$ and $\left.4.6 \mathrm{~Hz}, 1 \mathrm{H}, \mathrm{C}_{3},{ }_{-} \mathrm{H}\right)$, $1.06\left(\mathrm{~d}, J=6.5 \mathrm{~Hz}, 3 \mathrm{H}, \mathrm{CH}_{3}\right), 2.24\left(\mathrm{~m}, 1 \mathrm{H}, \mathrm{C}_{2},{ }_{-} \mathrm{H}\right), 2.72(\mathrm{dd}$, $J=13.2$ and $\left.8.2 \mathrm{~Hz}, 1 \mathrm{H}, \mathrm{C}_{1}, \cdot-\mathrm{H}\right), 2.90(\mathrm{dd}, J=13.2$ and $6.2 \mathrm{~Hz}$, $\left.1 \mathrm{H}, \mathrm{C}_{1},,-\mathrm{H}\right), 3.96\left(\mathrm{~s}, 3 \mathrm{H}, \mathrm{OCH}_{3}\right), 7.18(\mathrm{dd}, J=8.9$ and $1.6 \mathrm{~Hz}$, $\left.1 \mathrm{H}, \mathrm{C}_{6},-\mathrm{H}\right), 7.38\left(\mathrm{t}, J=7.3,1 \mathrm{H}, \mathrm{C}_{10}-\mathrm{H}\right), 7.45\left(\mathrm{~s}, 1 \mathrm{H}, \mathrm{C}_{6}-\mathrm{H}\right)$, $7.49\left(\mathrm{~m}, 2 \mathrm{H}, \mathrm{C}_{9}-\mathrm{H}\right.$ and $\left.\mathrm{C}_{11}-\mathrm{H}\right), 7.71\left(\mathrm{~d}, J=7.5 \mathrm{~Hz}, 2 \mathrm{H}, \mathrm{C}_{8}-\mathrm{H}\right.$ and $\left.\mathrm{C}_{12}-\mathrm{H}\right), 7.83\left(\mathrm{~d}, J=9.1 \mathrm{~Hz}, 1 \mathrm{H}, \mathrm{C}_{7},-\mathrm{H}\right), 8.49(\mathrm{~d}, J=2.0 \mathrm{~Hz}$, $\left.1 \mathrm{H}, \mathrm{C}_{2}-\mathrm{H}\right), 11.54(\mathrm{~s}, 1 \mathrm{H}, \mathrm{OH}) ;{ }^{13} \mathrm{C}-\mathrm{NMR}\left(\mathrm{CDCl}_{3}\right): 0.84\left(\mathrm{CH}_{3}\right)$, $1.87\left(6 \mathrm{CH}_{3}\right), 22.49\left(\mathrm{CH}_{3}\right), 26.18$ (C-3”), 29.28 (C-2”), 41.27 (C-1”), 94.22 (C-4'), 116.96 (C-2), 118.43 (C-7'), 122.76 (C6'), 126.81 (C-8 and C-12), 128.73 (C-9 and C-11), 131.96 (C5), 132.23 (C-1), 138.96 (C-7a'), 140.24 (C-7), 143.88 (C-3a'), 147.26 (C-4), 159.72 (C-5). Anal. Calcd for $\mathrm{C}_{30} \mathrm{H}_{43} \mathrm{~N}_{3} \mathrm{O}_{4} \mathrm{Si}_{3}$ : C, 60.67; H, 7.30; N, 7.07. Found: C, 60.89; H, 7.02; N, 7.18.

\section{References}

1 Hockberger P E, A history of ultraviolet photobiology for humans, animals and microorganisms, Photochem. Photobiol. 76 (2002), no. 6, 561-579, DOI 10.1562/0031-8655(2002)076<0561:AHOUPF>2.0.CO;2.

2 Clydesdale G J, Dandie G W, Muller H K, Ultraviolet light induced injury: Immunological and inflammatory effects, Immun. Cell Biology 79 (2001), 547-568, DOI 10.1046/j.1440-1711.2001.01047.x.

3 Ferrini R I, PerIman M, Hill L, Skin protection from ultraviolet light exposure, American college of preventive medicine, practice policy statement, Am. J. Prev.Medicine 14 (1998), 1-7.

4 Moan J, Dahlback A, The relationship between skin cancers, solar radiation and ozone depletion, Brit.J. Cancer, posted on 1992, 916-921, DOI 10.1038/bjc.1992.192, (to appear in print).

5 Kumler W D, Daniels T. C, Sunscreen compounds, J. Pharm. Sci. 37 (2006), 474-476, DOI 10.1002/jps.3030371111.

6 Cadet J, Sage E, Douki T, Ultraviolet radiation-mediated damage to cellular DNA, Mutat. Res. 571 (2005), 3-17, DOI 10.1016/j.mrfmmm.2004.09.012.

7 Ager N. S, Holliday G. A, Barnetson R. S, The basal layer in human squamous tumors harbors more UVA than UVB fingerprints mutations: a role for UVA in human skin carcinogenesis, Proc. Natl. Acad. Sci. USA 101 (2004), 4954-4959, DOI 10.1073/pnas.0401141101.

8 Rastogi S C, UV filters in sunscreen products - a survey, Contact Dermatitis 46 (2002), 348-351, DOI 10.1034/j.1600-0536.2002.460605.x.

9 Antoniou C, Kosmadaki M G, Stratigos A J, Katsambas A D, Sun- 
screens - what's important to know, J. Eur.Acad. Dermetol. Venereol. 22 (2008), 1110-1118, DOI 10.1111/j.1468-3083.2007.02580.x.

10 Chudoba C, Riedle E, Pfeiffer M, Elsaesser T, Vibrational coherence in ultrafast excited state proton transfer, Chem. Phys. Lett. 263 (1996), 622-628, DOI 10.1016/S0009-2614(96)01268-7.

11 Cawford J. C, 2-2(Hydroxy-phenyl)-2H-benzotriazole ultraviolet stabilizers, Prog. Polym. Sci. 24 (1999), 7-43, DOI 10.1016/S0079-6700(98)000124.

12 Fournier T, Pommeret S, Mialocq J.-C, Deflandre A, Rozot R, Fentosecond laser studies of excited state intramolecular proton transfer in an ultraviolet-filter molecule, Chem. Phys.Lett. 325 (2000), 171-175, DOI 10.1016/S0009-2614(00)00629-1.

13 Otterstedt J-E A, Photostability and molecular structure, J. Chem. Phys. 58 (1973), 5716-5725, DOI 10.1063/1.1679196.

14 Farkas R, Lhiaubet-Vallet V, Corbera J, Törincsi M, Gorchs O, Trullas C, Jiménez O, Miranda M A, Novak L, Synthesis of new 2(2'-hydroxyaryl)benzotriazoles and evaluation of their photochemical behavior as potential UV-filters, Molecules 15 (2010), 6205-6216, DOI 10.3390/molecules15096205.

15 Farkas R, Törincsi M, Kolonits P, Alonso J. O, Novak L, One-pot synthesis of benzotriazoles and benzotriazole 1-oxides by reductive cyclization of o-nitrophenylazo compounds with benzyl alcohol, Heterocycles 78 (2009), 2579-2588, DOI 10.3987/COM-09-11759.

16 Farkas R, Törincsi M, Kolonits P, Fekete J, Alonso O J, Novak L, Simultaneous displacement of a nitro group during coupling of diazotized o-nitroaniline with phenols., Cent. Eur. J. Chem. 8 (2010), 300-307, DOI 10.2478/s11532-009-0150-8.

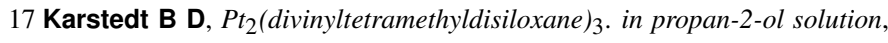
4 June 1974. U.S. Patent 3,814,720. 